\title{
Topological Localized states the time delayed Adler model: Bifurcation analysis and interaction law
}

\author{
L. Munsberg, ${ }^{1,2}$ J. Javaloyes, ${ }^{3}$ and S. V. Gurevich ${ }^{1,2,3, a)}$ \\ ${ }^{1)}$ Institute for Theoretical Physics, University of Münster, Wilhelm-Klemm-Str. 9, D-48149 Münster, \\ Germany \\ ${ }^{2)}$ Center for Nonlinear Science (CeNoS), University of Münster, Corrensstrasse 2, D-48149 Münster, \\ Germany \\ ${ }^{3)}$ Departament de Física $\&$ IAC-3, Universitat de les Illes Balears, C/ Valldemossa km 7.5, 07122 Mallorca, \\ Spain
}

The time-delayed Adler equation is arguably the simplest model for an injected semiconductor laser with coherent injection and optical feedback. It is able to reproduce the existence of topological localized structures (LSs) and their rich interactions. In this paper we perform the first extended bifurcation analysis of this model and we explore the mechanisms by which LSs emerge. We also derive the effective equations governing the motion of distant LSs and we stress how the lack of parity in time-delayed systems leads to exotic, nonreciprocal, interactions between topological localized states.

\section{INTRODUCTION}

Localized structures (LSs) appear in driven dissipative nonlinear systems and they can be observed in a variety of complex systems ${ }^{1-5}$. They are attractors of the dynamics, i.e. stable solutions towards which the system converges from a wide set of initial conditions ${ }^{6}$. In Optics, LSs are usually envisioned as light pulses in time or localized beams in space, $\operatorname{see}^{7-9}$ for reviews, and one distinguishes between systems in which the LSs are locked to an external injection beam from the ones that possess a phase invariance. The former situation leads to the so-called cavity solitons ${ }^{10,11}$ observed either in the transverse plane of broad area amplifiers ${ }^{12}$ or in the temporal output of fibers ${ }^{13,14}$. In phase invariant situations, spatial diffractive autosolitons were predicted and observed either in cavities composed of a gain medium coupled to a saturable absorber ${ }^{15-17}$ or to an external diffraction grating $^{18}$. Temporal localization was also achieved in passively mode-locked lasers operated in the long cavity regime $^{19}$ and it was demonstrated that a similar setup could also lead to full thee-dimensional spatio-temporal localization $^{20}$.

Spatial and temporal LSs of light have often been analyzed using similar theoretical frameworks. However, while space is isotropic, temporal dynamics usually exhibits a symmetry breaking due to the causal response of the active medium. The dynamics of temporal LSs may break the action-reaction principle, an effect that was discussed for instance in the framework of modelocking ${ }^{21,22}$. Building upon the strong analogies between spatially extended and time-delayed systems ${ }^{23-25}$ (TDSs), the latter have been proposed for generating temporal LSs, see ${ }^{26}$ for a recent review. In TDSs, propagation and nonlinearity occur in well separated stages, which is at variance with distributed systems, such as, e.g., the nonlinear Schrödinger equation governing light

a)Electronic mail: gurevics@uni-muenster.de propagation in fibers. In most cases, LSs appear in TDSs as mutually independent light peaks ${ }^{19,27}$. However, topological LSs were also predicted and observed in semiconductor lasers; They exist either as $2 \pi$ kinks in the polarization orientation ${ }^{28}$ or in the phase of the optical field $^{29}$. In this latter case, it was shown that a simple time-delayed model for the phase of the lasing field is able to reproduce the results obtained in ${ }^{29,30}$. Despite its simplicity, this model contains the effects of optical injection, frequency locking and time-delayed feedback and can be termed the time-delayed Adler equation.

In this manuscript, we perform an extended analysis of the delayed Adler equation using the path continuation package dde-biftool ${ }^{31}$ and asymptotic analysis. In particular, we provide the effective equations of motion for distant, weakly interacting, topological LSs and we give the conditions under which repulsive and attractive forces can give rise to stable molecules. Finally, we show that the interaction between LSs are not reciprocal, a feature typical of system with broken parity symmetry.

\section{MODEL}

Topological LSs can be obtained by combining two elements. The first is a semiconductor laser with coherent optical injection operated in the so-called "excitable" regime $^{32}$. In this regime, the phase of the semiconductor laser is stably locked to the external forcing. Upon small perturbations, the phase, that evolve on a circle, relaxes (e.g., clockwise) exponentially to its equilibrium state. However, when responding to a sufficiently large external perturbation, the phase performs a $2 \pi$ anti-clockwise rotation, after which the system locks again to the external forcing. This mechanism leads to a simple scenario of excitability. To this injected laser system, we add a delayed feedback loop. Here, the delayed feedback plays the role of the extended (spatial) degree of freedom ${ }^{24}$ in which multiple independently addressable topological LSs can be stored and regenerated indefinitely ${ }^{29}$. Each $2 \pi$ phase structures, embedded in a homogeneously locked back- 
ground field, propagate into the external feedback loop, similarly to the sine-Gordon solitons ${ }^{33}$. When these kinks come back into the semiconductor laser they act as triggers for new excitable dynamics and, hence they get regenerated. By assuming small injection and optical feedback, as well as a small detuning between the injection field and the laser natural frequency, a multiple time scale analysis yields the time-delayed Adler equation for the phase evolution $\theta(t)$

$$
\dot{\theta}=\Delta-\sin \theta+\chi \sin [\theta(t-\tau)-\theta-\psi],
$$

where the dot denotes the time derivative with respect to a slow time $t, \Delta$ the ratio and of the detuning between the injection and the laser normalized to the injection field amplitude, $\chi>0$ the ratio of the feedback rate and of the amplitude of the injection field, while the parameter $\psi$ is directly related to the feedback phase, see Sup. Mat. in $^{29}$ for more details. In the absence of feedback $(\chi=0)$, Eq. (1) becomes an Adler equation that describes e.g., a time evolution of the phase difference of two weakly coupled oscillators with a small detuning in their frequencies. Here, one notices the presence of saddle node on a circle bifurcation that arises at $\Delta_{ \pm}= \pm 1$; It is in the vicinity of $\Delta_{+}$that the Adler equation exhibits excitability. Temporal LSs are found in the long time delay limit ${ }^{34}$ in which the excitable orbit duration is shorter than the time delay. From the strict point of view of TDSs, temporal LSs are peculiar periodic solutions that consists in a localized waveform, that is independent of the precise value of $\tau$, if $\tau$ is large enough, embedded into an arbitrary large chunk of the homogeneous solution. Recently, a complete classification of the spectrum of TDSs into interface and pseudo-continuous spectrum was achieved ${ }^{34}$ and the link between LSs periodic orbits with homoclinic solutions was clarified.

\section{RESULTS}

We start the analysis by searching the steady states of Eq. (1) that are defined by

$$
0=\Delta-\sin \theta-\chi \sin \psi
$$

which is solved by

$$
\begin{aligned}
& \theta_{s}=\arcsin (\Delta-\chi \sin \psi)+2 \pi n, \quad n \in \mathbb{Z}, \\
& \theta_{u}=\pi-\arcsin (\Delta-\chi \sin \psi)+2 \pi n . \quad n \in \mathbb{Z} \text {. }
\end{aligned}
$$

making that $\theta_{s}$ (resp. $\theta_{u}$ ) belongs to the right (resp. left) half of the unit circle, i.e. $\theta_{s} \in\left[-\frac{\pi}{2}, \frac{\pi}{2}\right]$ and $\theta_{u} \in\left[\frac{\pi}{2}, \frac{3 \pi}{2}\right]$. The linear stability analysis information for the surroundings of the steady states is obtained setting $\theta_{s, u}+\varepsilon \delta$ with $\varepsilon \ll 1$, which yields

$$
\begin{aligned}
& \dot{\delta}=A \delta(t)+B \delta(t-\tau), \\
& A=-\cos \theta_{s, u}-\chi \cos \psi, \\
& B=\chi \cos \psi,
\end{aligned}
$$

where $A$ and $B$ are the instantaneous and delayed Jacobian matrices of the linearized problem evaluated at the steady state. The characteristic equation is found setting $\delta(t)=\delta_{0} \exp (\lambda t)$, leading to the transcendental equation

$$
\lambda=A+B e^{-\lambda \tau} .
$$

Note that without delayed feedback $(\chi=0)$ the eigenvalues $\lambda_{s, u}$ are given by:

$$
\lambda_{s, u}=-\cos \theta_{s, u}
$$

leading to $\lambda_{s}<0$ and $\lambda_{u}>0$. Therefore without delay the solution $\theta_{s}$ is always stable and $\theta_{u}$ is always unstable. Furthermore, for $\Delta_{ \pm}= \pm 1$ both steady states coincide at:

$$
\theta_{ \pm}= \pm \frac{\pi}{2}+2 \pi n, \quad n \in \mathbb{Z}
$$

and disappear in a saddle-node bifurcation for $|\Delta|>1$.

Considering the influence of the delay, the steady states only exist for:

$$
\Delta-\chi \sin \psi \in[-1,1]
$$

leading to the boundaries of existence for the steady states as

$$
\begin{aligned}
& \Delta_{s n}^{+}=1+\chi \sin \psi, \\
& \Delta_{s n}^{-}=-1+\chi \sin \psi,
\end{aligned}
$$

which effectively shifts the saddle-node bifurcation to higher values of $\Delta$ for $\psi \in[0, \pi]$ and to lower values for $\psi \in[\pi, 2 \pi]$. The amplitude of the shift is given by $\chi$.

The characteristic equation (5) can not be solved analytically for $\chi \neq 0$ as it is transcendental. However solutions for $\lambda$ can be found using the Lambert $W$ functions ${ }^{35}$

$$
\lambda_{n}=A+\frac{1}{\tau} W_{n}\left(B \tau e^{-A \tau}\right) .
$$

The resulting number of eigenvalues is infinite because of the infinite number of branches of the Lambert $W$ functions denoted $W_{n}$ with $n \in \mathbb{Z}$. In the limit of long delays the infinite number of eigenvalues accumulate over a quasi-continuous spectrum ${ }^{36}$. By expanding the eigenvalues in real and imaginary part $\lambda=\frac{\alpha}{\tau}+i \beta$ in Eq. (5) one obtains

$$
\alpha=\frac{1}{2} \ln \left(\frac{B^{2}}{A^{2}+\beta^{2}}\right) .
$$

In figure 1 the quasi-continuous spectrum as well as the leading eigenvalues obtained from Eq. (9) are plotted. One can observe that even for small values of the delay time $\tau=5$ (see the panel (a)), the quasi-continuous spectrum is a good approximation for the exact eigenvalues obtained from Eq. (9). For larger values of $\tau$ the distance between the discrete eigenvalues is smaller leading to an increasing number of relevant eigenvalues. 

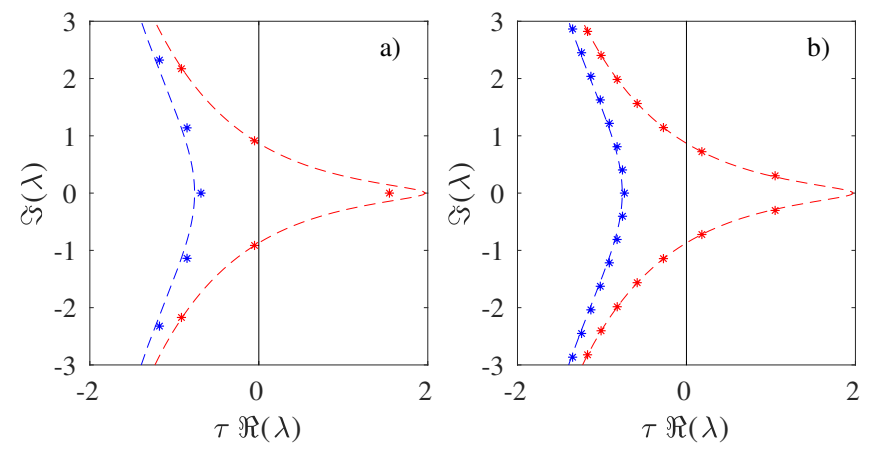

Figure 1. Eigenvalues $\lambda_{s}$ (blue) and $\lambda_{u}$ (red) of Eq. (5) corresponding to the steady states $\theta_{s, u}$ for (a) $\tau=5$ and (b) $\tau=15$. The dashed lines show the quasi-continuous spectrum (10) while the stars represent the exact solutions given by the Lambert $\mathrm{W}$ functions (9). Other parameters are $(\Delta, \chi, \psi)=(0.5,1,0.5)$.

For large delays one can estimate the instability threshold for the steady states by calculating the set of parameters leading to a crossing of the quasi-continuous spectrum with the imaginary axis. Depending on whether the leading discrete eigenvalues are real or a set of complex conjugates, the occurring bifurcation is a saddlenode (SN) or Andronov-Hopf (AH) bifurcation. In our case, the first $\mathrm{AH}$ point is given by $\alpha=0$ that we approximate in the long delay limit as $\beta \sim 1 / \tau=0$. Because the maximum real part of Eq. (10) is obtained for a vanishing imaginary part, this leads to the relation $A^{2}=B^{2}$. The first two borders obtained by setting $A=-B$ correspond to the SN bifurcation identified previously in Eqs. ((3),(4)) while the branch $A=B$ yields the following borders of stability at which the steady state gets AH unstable; for the solutions $\theta_{s}$ and $\theta_{u}$, these borders have an explicit expression that reads

$$
\begin{aligned}
& \Delta_{s}^{ \pm}= \pm \sqrt{1-4 \chi^{2} \cos ^{2} \psi}+\chi \sin \psi, \cos \psi<0 \\
& \Delta_{u}^{ \pm}= \pm \sqrt{1-4 \chi^{2} \cos ^{2} \psi}+\chi \sin \psi, \cos \psi>0
\end{aligned}
$$

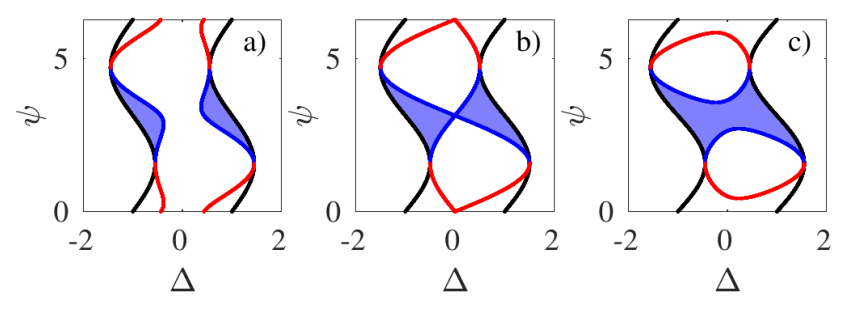

Figure 2. Dependence of AH unstable regime, shaded in blue, on feedback strength $\chi$ in the long delay limit (10). Blue lines show the $\mathrm{AH}$ instabilities of $\theta_{s}$, while red lines show the $\mathrm{AH}$ instabilities of $\theta_{u}$ and black lines correspond to the SN bifurcation of both steady states. Feedback strength changes from (a) $\chi=0.45$, (b) $\chi=0.5$ to (c) $\chi=0.55$.

The area in which the steady state $\theta_{s}$ is $\mathrm{AH}$ unstable is especially important because this results in an oscillating background for LSs. The change of size and shape of the
AH unstable area as a function of the feedback strength $\chi$ was investigated in Fig. 2; the four borders resulting from Eqs. (11),(12) are plotted for different values of $\chi$ and the region of instability of $\theta_{s}$ is colored in blue. For low feedback strengths, there are two areas in which the steady state $\theta_{s}$ is $\mathrm{AH}$ unstable and they are close to the SN bifurcation between $\psi=\pi / 2$ and $\psi=3 \pi / 2$, see Fig. 2(a). With increasing feedback strength, these areas stretch further away from the SN border, until they meet at a critical point defined by $\left(\Delta_{c}, \chi_{c}, \psi_{c}\right)=(0,1 / 2, \pi)$ as shown in Fig. 2(b). For even bigger feedback strengths of $\chi>\chi_{c}$ the two areas merge into one area of instability (cf. Fig. 2(c)).
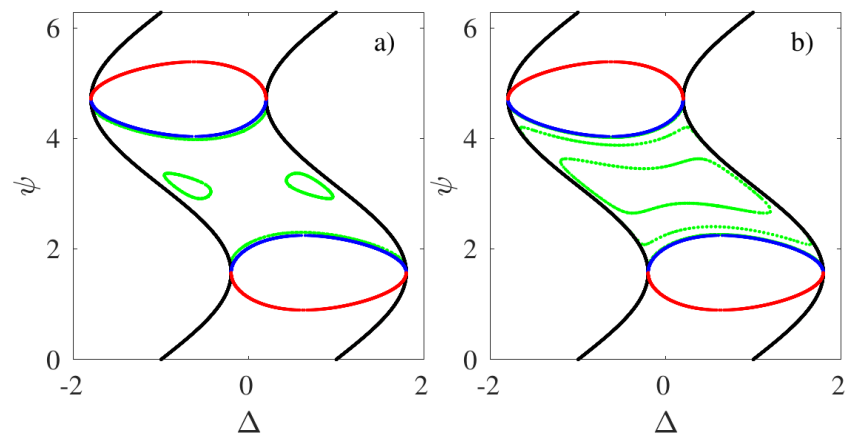

Figure 3. Borders of the AH instability obtained from the path-continuation (green) for (a) $\tau=10$ and (b) $\tau=20$, respectively. Steady state instabilities in the long delay limit are shown in black (SN) blue $\left(\mathrm{AH}\right.$ of $\left.\theta_{s}\right)$ and red (AH of $\left.\theta_{u}\right)$.

Using the dde-biftool ${ }^{31}$ framework it is possible to follow the steady state $\theta_{s}$ while varying the parameters $\Delta$ and $\psi$. If there an AH bifurcation occurs, it is possible to follow the $\mathrm{AH}$ bifurcation point in the $(\Delta, \psi)$ plane. Since several pairs of eigenvalues can cross the imaginary axis, this method allows obtaining several curves corresponding to the crossing of the imaginary axis. In Fig. 3 the results of the continuation are shown for two different time delays $\tau$. For each $\tau$ one can observe an AH border close to but not exactly at the border obtained from the long delay limit approximation, corresponding to the crossing of the first pair of complex conjugate eigenvalues with the imaginary axis. For small delay times as shown in Fig.3(a) for $\tau=10$, the regions where a second pair of complex conjugate eigenvalues have a positive real part form two separate small ovals centered around $\psi=\pi$. With increasing delay time the distance between the discrete eigenvalues becomes smaller, while they still follow the quasi-continuous spectrum which is independent on the delay time. This leads to a larger region of instability for each pair of complex conjugate eigenvalues crossing the imaginary axis. In the case of Fig. 3(b), the larger delay $\tau=20$ leads to a merging of the two small regions of secondary instability into one large region. Notice that a good approximation of these additional AH lines, and more generally of the complex eigenvalue spectrum, can be obtained setting $\beta_{n} \sim 2 \pi\left(n+\frac{1}{2}\right) / \tau$ in Eq. (10) to find $\alpha_{n}$. Setting $\alpha_{n}$ allows finding the green curves depicted 
in Fig. 3. We note that the first $\mathrm{AH}$ bifurcation appears with frequency $\beta_{1} \sim \pi / \tau$ which corresponds to a period two regime, characteristic of time delayed systems.
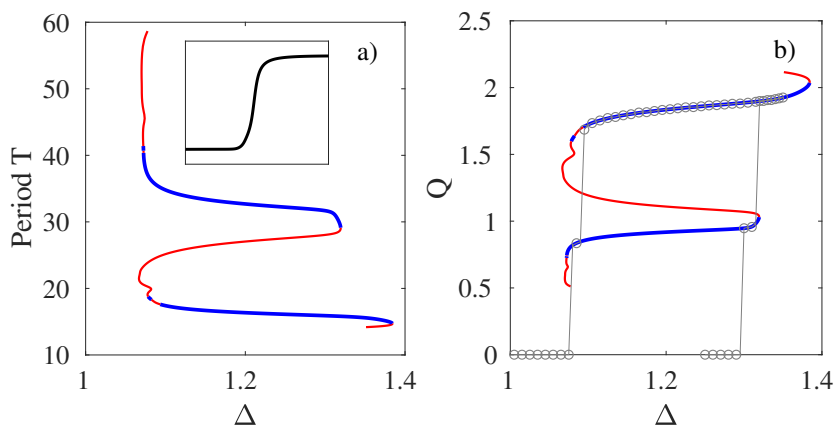

Figure 4. Bifurcation diagram of periodic solutions of Eq. (1). Here blue bold lines show stable solutions while red thin lines show unstable ones. In (a) the y-axis displays the period $T$ of the solution while in (b) the topological charge $Q$ (13) is shown on the y-axis. The inset in (a) represents the exemplary periodic solution profile. The black crosses in (b) show results of a direct numerical integration with varying detuning $\Delta$. Parameters are $\chi=0.3, \tau=30$ and $\psi=1.4$.

In the region surrounding the SN bifurcations the system is excitable. A small perturbation from the stable state $\theta_{s}$ decays exponentially because the system is linearly stable. If however the system is close to the SN bifurcation, the states $\theta_{s}$ and $\theta_{u}$ are close. In this case, a finite size perturbation can excite the system beyond the linearly unstable state $\theta_{u}$ leading to a trajectory towards $\theta_{s}+2 \pi$ instead of relaxing back directly towards $\theta_{s}$. This means that a small perturbation of a stable state can lead to a large orbit in phase space. If we now introduce time delay into this excitable system, one excitation at the time $t-\tau$ can lead to another excitation at time $t$ resulting in a periodic repetition of excitations with an approximate period of the delay time $\tau$. In the inset of figure 4 (a) an example of a periodic orbit from $\theta_{s}$ to $\theta_{s}+2 \pi$ is shown. In the following, we shall concentrate our attention on positive (upward) kinks. Downward (anti-kinks) can be deduced from the kink regimes by using the symmetry of Eq. .(1) $(\theta, \Delta, \psi) \rightarrow-(\theta, \Delta, \psi)$. Hence if kinks are found for parameters $(\Delta, \chi, \psi)=\left(\Delta^{*}, \chi^{*}, \psi^{*}\right)$, identical anti-kinks exist at $(\Delta, \chi, \psi)=\left(-\Delta^{*}, \chi^{*},-\psi^{*}\right)$.

We further investigate these regenerative excitable orbits with the help of dde-biftool. They are implemented as $T$-periodic orbits, going from from $\theta_{s}$ towards $\theta_{s}[2 \pi]$. We note that $[2 \pi]$ modulo operator is implemented automatically in the recent versions of dde-biftool. The periodic solutions were then continued in $(\Delta, \chi, \psi)$ and $T$ while adjusting the profile of $\theta(t)$ on an adaptive grid. The resulting branch of solutions is shown in Fig. 4(a). One can observe two stable regions, one having a period slightly above $\tau=30$ and the other one having a period slightly above $\frac{\tau}{2}=15$. To achieve a better visualization of the periodic solutions, the topological charge $Q$, corresponding to the number of $2 \pi$-phase differences per time delay $\tau$ is introduced:

$$
Q=\frac{\tau}{T_{1}-T_{0}} \int_{T_{0}}^{T_{1}} \frac{\mathrm{d} \theta}{2 \pi}
$$

For the parameters used in Fig. $4(\psi=1.4, \chi=0.3)$, the steady state $\theta_{s}$ vanishes at $\Delta=1.296$ but the continuation clearly shows stable periodic solutions for values of $\Delta>1.296$. To investigate this region further, a direct numerical integration was performed starting with the state $\theta_{s}$ at $\Delta=1.25$. After integrating for a time long enough to ensure stability of the result, the parameter $\Delta$ was changed and the next step of integration was performed starting with the result of the former integration. First $\Delta$ was increased up to 1.35 which lies outside of the range of existence of $\theta_{s}$. After reaching $\Delta=1.35$ the process was continued in the other direction till $\Delta=1$. For each step the topological charge $Q$ was calculated using Eq. (13). In Fig. 4(b) the topological charge $Q$ is shown for the results of the time integration and the continuation. One can observe that the system starts and stays in the state $\theta_{s}$ which has a topological charge of 0 until around $\Delta=1.296$ the system jumps to the stable branch with $Q \approx 1$. At the point where the stable periodic solution with $Q \approx 1$ disappears in a SN bifurcation the system jumps to the stable solution with $Q \approx 2$ which is stable up to values of $\Delta>1.35$. In the reverse direction where $\Delta$ is decreased from $\Delta=1.35$ to $\Delta=1$ one can observe a hysteresis because there is a region ranging from $\Delta \approx 1.1$ to $\Delta \approx 1.3$ where all three solutions with $Q=0, Q \approx 1$ and $Q \approx 2$ are stable. After reaching $\Delta \approx 1.1$ the system falls back to the solution with $Q \approx 1$ which also gets unstable for $\Delta \approx 1.075$ resulting in the system falling back to $\theta_{s}$ with $Q=0$.

A global way of displaying the instabilities of the periodic solutions such as torus and period-doubling bifurcation consists in representing the charge $Q$ as a function of $\Delta$ and $\psi$. To this aim, several branches of periodic solutions were calculated for equidistant values of $\psi$. The resulting point cloud was then interpolated into a two-dimensional surface of periodic solutions. There is certainly a loss of accuracy in this interpolation but the resulting surface is only used for illustrative purposes. In Fig. 5 one perspective of this surface is plotted in the three-dimensional representation, as well as the background instabilities of the steady state at $Q=0$ and the three types of instabilities for the periodic solutions. A video of the full thee-dimensional structure can be found in the Supplementary Material. Here one can clearly see that the stable regions of periodic solutions form one connected surface that increase in $Q$ by 1 if one increases $\phi$ gradually by $2 \pi$. This band of stable solutions is in some cases interrupted by the torus or period doubling bifurcations leading to unstable regions inside the stable surface. Since all branches of periodic solutions that were continued in $\Delta$ for a specific value of $\psi$ represent a cut of this surface, those regions of instability lead to a splitting of the stable regions as seen in Fig. 4. 


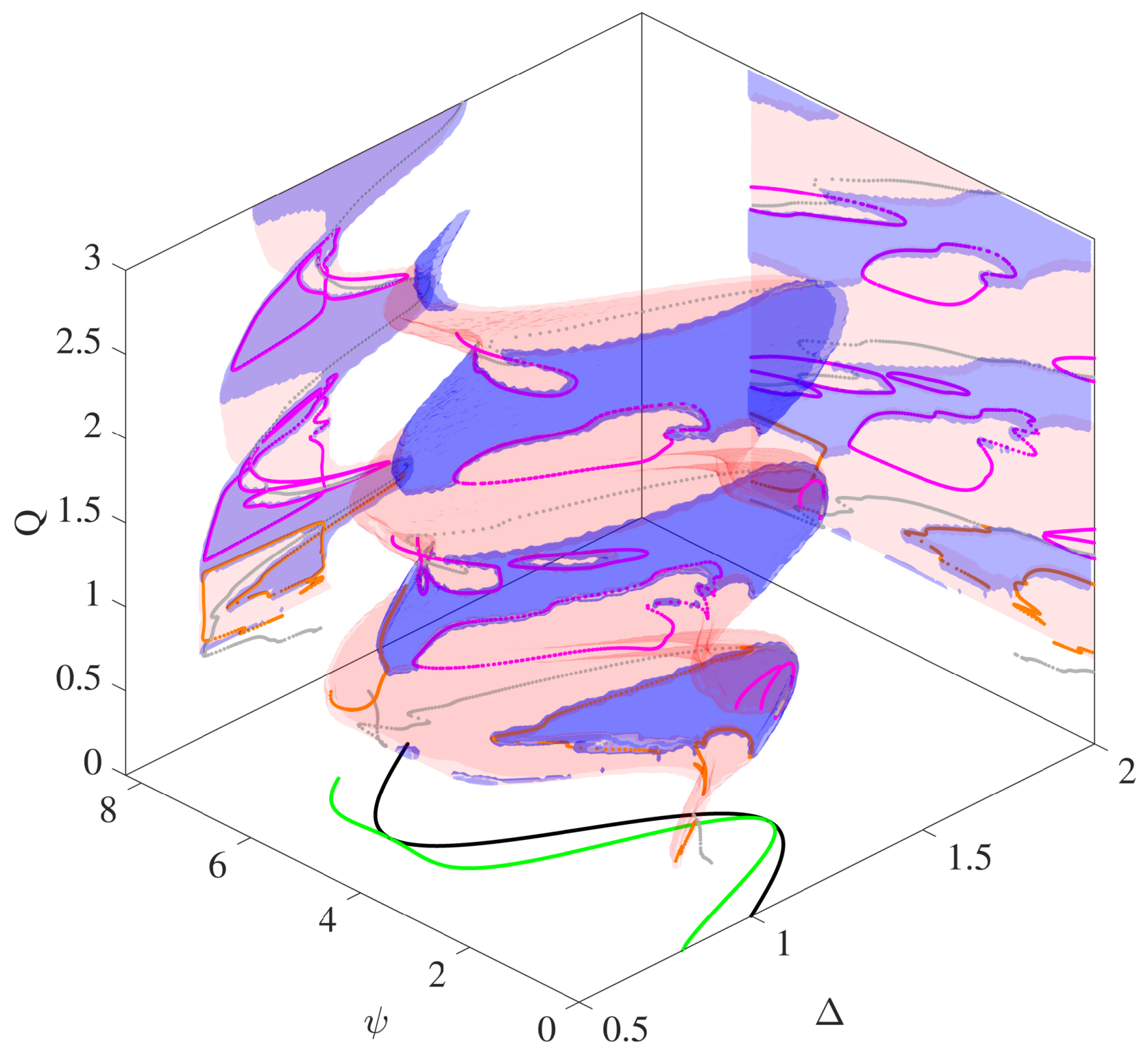

Figure 5. Three-dimensional representation of the bifurcation structure of periodic solutions. Blue and red surfaces represent stable and unstable solutions. Grey, orange and magenta lines show borders of fold, period doubling and torus instabilities respectively. The long delay instabilities of the steady states are included at $Q=0$ (black and green lines) as a reference. In the background a two-dimensional projection on to the $(Q-\Delta)$ and $(Q-\psi)$ planes is shown. Parameters are $\chi=0.3$ and $\tau=30$.

An interesting kind of periodic solution are those, whose period $T$ are diverging and that can be much greater than the delay $\tau$. These solutions have a small value of $Q$ and therefore approach the steady state for which $Q=0$. In Fig. 5 these solutions were left out due to computation time. We investigate those solutions with low topological charge $Q$ to learn more about their connection to the branch of periodic solutions and to the steady state. One possible connection involves the $\mathrm{AH}$ instability of the steady state $\theta_{s}$. In Fig. 3 we indeed showed that several AH instabilities appear increasing the time delay $\tau$. We could therefore prepare the system in a parameter regime such that there is a stable steady state $\theta_{s}$ for small delay values and make it $\mathrm{AH}$ unstable by increasing the delay time. With a further increase in $\tau$ the amplitude of the libration grows up to the point that it induces the nucleation of as a fully developed rotating solution. Notice that these solutions can not be considered as LSs since the latter need a stable background. Since an AH instability leads to small amplitude peri- 


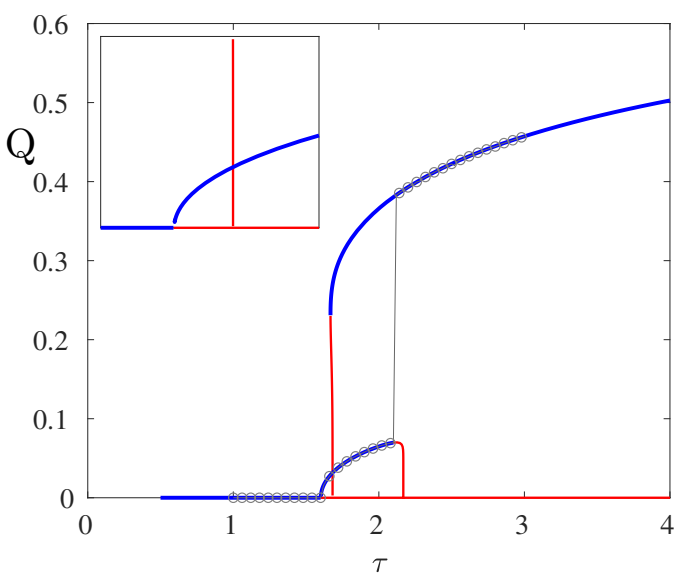

Figure 6. Bifurcation diagram in $(Q-\tau)$ plane, showing branches of rotating solutions and librations around the steady state $\theta_{s}$. Unstable and stable solutions are indicated as red thin lines and blue bold lines, respectively. Results obtained by direct numerical integration with varying delay $\tau$ are also shown as a gray circles. Parameters are $(\Delta, \chi, \psi)=(0.7,1,2.84)$

odic oscillations and due to the frequent use of the term periodic solutions for the LSs, we will constrict the use of the term rotating solutions to LSs and, more generally, to the unlocked solutions where the temporal variations of the phase are unbounded. We will refer to the small oscillations arising from the $\mathrm{AH}$ instabilities as librations.

In order to compare the branches corresponding to rotations and librations in a single bifurcation diagram, we have to adjust our measure $Q$ since using the definition given in Eq. (13) both the steady state and the librations would have a topological charge of 0 . While this accurately represents the number of $2 \pi$ phase flips that happen in a period $T$, we want to observe the transition between those states. For this reason a different measure was used to display the different branches in Fig. 6:

$$
Q=\frac{\delta \theta}{2 \pi} \frac{\tau}{T}
$$

defining $\delta \theta$ as the amplitude of the phase variation over the period T. In Fig. 6 the three branches of solutions are displayed with this new measure on the vertical axis. One can clearly see the stable oscillating branch emerging from the $\mathrm{AH}$ bifurcation point of the steady state at $\tau_{A H} \approx 1.6$. The branch of rotating solutions, however, does not seem to emerge from the branch of librations, but to connect with the homogeneous solution using this measure. One notices that the branch of rotating solutions reaches values of $Q$ that are much lower than unity. With our definition of $Q$ given in Eq. 14, this is only possible if the period $T$ of the branch of LSs diverges, thereby indicating a global bifurcation connecting the rotating solution with the steady states.

A possible candidate would be a homoclinic bifurcation, however, the Adler equation without delay is some-
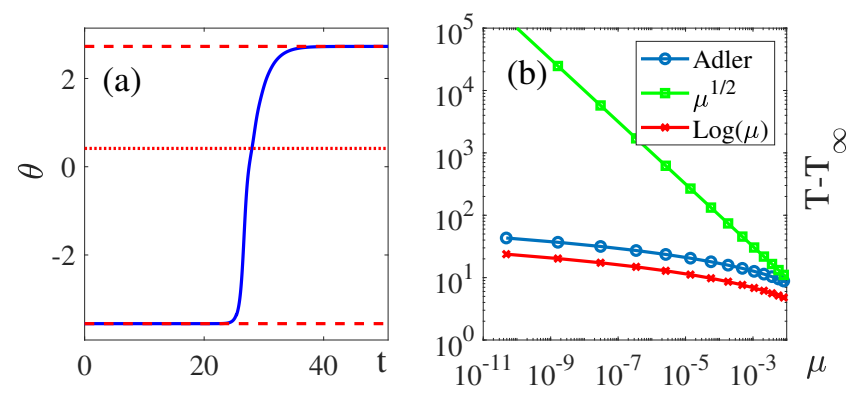

Figure 7. (a) Profile of the periodic solution (a) at $\tau=\tau^{*}$ that connects the unstable steady state $\theta_{u}$ (dashed lines) with itself. The solution $\theta_{s}$, that is AH unstable at this value of $\tau$ is marked by a dotted line. (b) Scaling of the period in the vicinity of the bifurcation point as a function of $\mu=\tau-\tau^{*}$ with $\tau^{*} \simeq 1.684511$. Parameters are $(\Delta, \chi, \psi)=(0.7,1,2.84)$.

times considered to be the normal form of the global saddle-node infinite period bifurcation (SNIPER) ${ }^{37}$. As such, one may expect the global bifurcation connecting the steady state and the periodic solution of the delayed Adler equation to be a SNIPER as well. The two possible bifurcations can be distinguished by the scaling of the period as a function of the distance $\mu$ to the bifurcation point, while approaching the latter. Figure 7(a) depicts a profile obtained as close as possible to the bifurcation point, using 150 collocation points and sixth order polynomials, that we approximate at $\tau^{*} \simeq 1.684511$, and for which the period is $T \sim 51$. One notices clearly that this rotating orbit connects the unstable steady state $\theta_{u}$ onto itself. Figure $7(\mathrm{~b})$ allows us to verify that the period scales with $\log \mu$ with $\mu=\left|\tau-\tau^{*}\right|$ indicating a homoclinic orbit. We noticed that the transition layer in Figure $7(\mathrm{a})$ is relatively smooth indicating that, considering the low values of $\tau$ used, $\theta(t-\tau)$ could be expanded in Taylor series. At second order in the truncation, Eq. 1 transforms into an ordinary differential equation for a forced, damped, nonlinear oscillator whose inertia and damping terms depend on $\tau$. It is in principle possible to search for specific values of $\tau$ at which infinite period solutions exist, leading to an approximation of $\tau^{*}$.

More surprisingly, we have found that the homoclinic bifurcation scenario is not always the reason behind the emergence of rotating solutions. This can be observed in Fig. 8(a) where we depict the bifurcation diagram including steady states, librations and rotating solutions for a set of parameters $(\Delta, \chi, \psi)=(0.1,1,2.84)$ for which the steady states are $\mathrm{AH}$ unstable but where the solutions are far from the SN bifurcation of steady states. The panels Fig. 8(b,c) display the profile of these period two (P2) solutions for which $T \sim 2 \tau$, which explains why $Q \sim 0.5$ for the rotating solution. It is well known that time delayed systems can give rise to such P2 regimes, see for instance $^{38,39}$ and reference therein.

We are interested, in particular, in understanding by which bifurcation scenario temporal LSs appear. In all the non-pathological cases explored, i.e. when the back- 

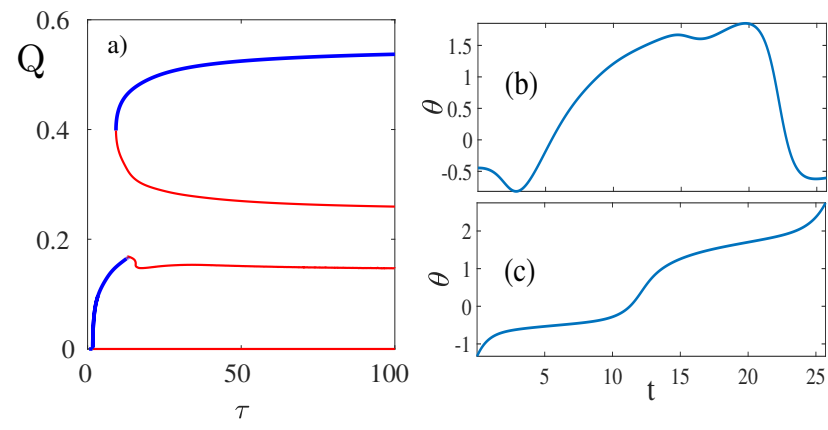

Figure 8. (a) Bifurcation diagram showing the branches of libration and of rotation and the unstable steady branch $\theta_{s}$ as a function of $\tau$. Parameters are $(\Delta, \chi, \psi)=(0.1,1,2.84)$ and the stability is indicated as red thin lines for unstable solutions and blue bold lines for stable solutions. (b) libration and (c) rotation are bistable at $\tau=12.87$ and both solutions have a period close to $2 \tau$.

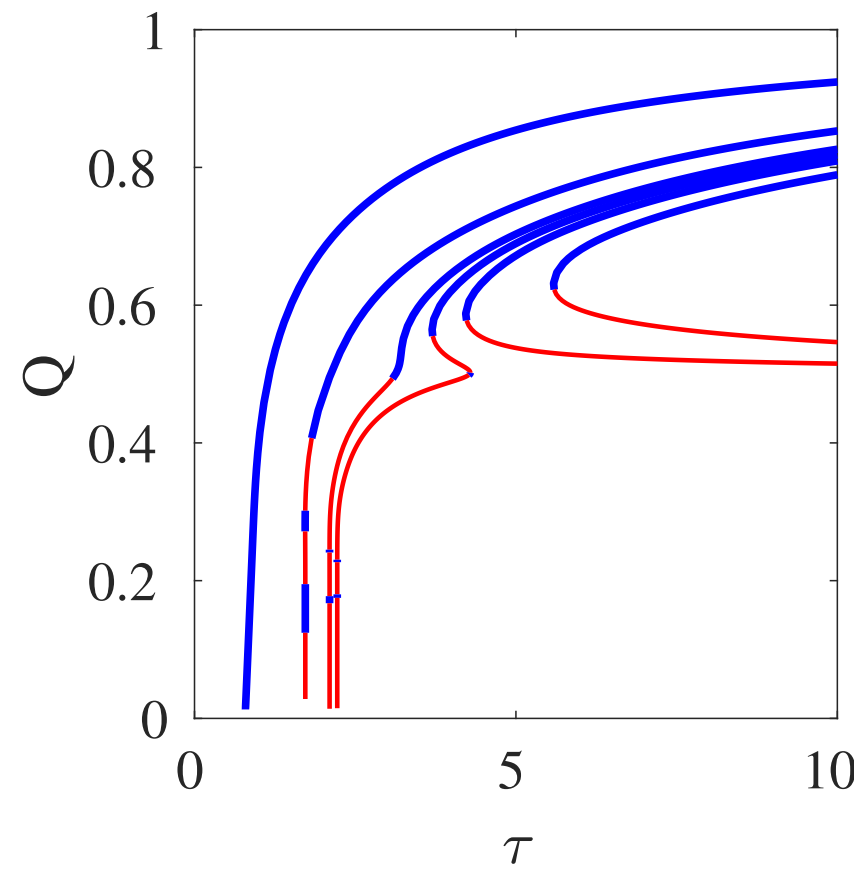

Figure 9. Bifurcation diagram showing the deformation of the branches of rotations with varying distance from the saddle node of steady states in a range of detuning $\Delta \in[0.7,2]$, while other parameters are $\chi=1$ and $\psi=\pi / 2$. The leftmost branches correspond to the larger values of $\Delta$ and the stability is indicated as red thin lines for unstable solutions and blue bold lines for stable solution. In all cases the steady solution $\theta_{s}$ is stable and the stable rotating orbits correspond to LSs, for large values of $\tau$.

ground solution is linearly stable, we have always found the LSs to appear from homoclinic bifurcations, with the period scaling as $\log \mu$, of from SN bifurcations of periodic solutions. We represent several branches of rotations for various values of $\Delta$ in Fig. 9. Multiple branches of periodic solutions were calculated for different distances
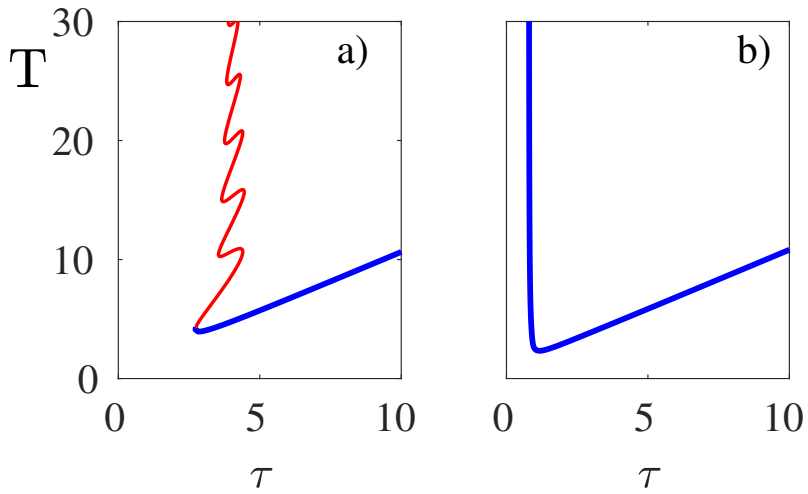

Figure 10. Branch of periodic solutions with $\tau$ as a free parameter for varying feedback phases calculated at the saddle node of steady states $\Delta=\Delta_{s n}^{+}$. (a) $(\Delta, \chi, \psi)=(1,1,0)$ shows snaking on the diverging branch. (b) $(\Delta, \chi, \psi)=(2,1, \pi / 2)$ shows no snaking. In both cases, the period does not scale with $\mu^{-1 / 2}$ or with $\log \mu$.

from the SN bifurcation of the steady states which is at $\Delta_{s n}^{+}=2$ for the given values of $\psi$ and $\chi$. A transition from a global bifurcation for the largest values of $\Delta$ towards a SN bifurcation of periodic solutions is clearly visible. Periodic solutions in the vicinity of the SN of the steady states show a global bifurcation while for larger distances of $\Delta$ with respect to $\Delta_{s n}^{+}$the branch deforms, while conserving the homoclinic period divergence at the branching point, and finally shows a regular saddle-node of limit cycle bifurcation for values of $\Delta<0.825$. The deformation of the branches in Fig. 9 indicates that a reconnection mechanism with other unstable branches of periodic solutions underlies the qualitative change in the bifurcation scenario.

Finally, we followed the evolution of the point at which the period diverges as a function of delay $\tau$ and feedback strength $\chi$ by calculating several branches in the limit case where the background solution $\theta_{s}$ is marginally stable. The detuning $\Delta$ was chosen such that the system is on the border of the steady state SN bifurcation, which is given by $\Delta=\Delta_{s n}^{+}$. Figure 10 shows the branches for two parameter sets. In both cases, the period diverges for small delay yet it does not scales with $\mu^{-1 / 2}$ or with $\log \mu$. It is particularly salient in Fig. 10(b), where the branch shows a snaking behavior. For larger delays, the solutions get more and more localized with a period that scales as $T=\tau+r$ with $r=\mathcal{O}(1)$. However, the background is marginally stable leading to solutions that can hardly be called LSs. They correspond to the pathological case discussed in ${ }^{34}$ Fig. 4.

\section{EFFECTIVE INTERACTION LAW}

We now turn our attention to the interaction law governing the relative motion of multiple LSs that can be embedded in a sufficiently long value of the time delay. Dis- 
tant LSs interact via their exponentially decaying tails. While in many salient examples the solitons are even functions, as e.g, those found as solutions of the Nonlinear Schrödinger, Ginzburg-Landau or Lugiato-Lefever equations, the left and right exponential tails are not necessarily identical in non-parity preserving systems. As such, the interactions between LSs may not be reciprocal and disobey with the action-reaction principle, as demonstrated recently in passively mode-locked laser ${ }^{21}$. For our analysis, it is convenient to factor out the value of the steady state $\theta_{s}$. We define $\theta(t)=\theta_{s}+\phi(t)$ in such a way that $\phi(t)$ can represents stable LSs from 0 to $2 \pi$. Then the right hand side of the original delayed Adler equation (1) transforms to

$$
\dot{\phi}=F\left(\phi, \phi^{\tau}\right)
$$

with

$$
F\left(\phi, \phi^{\tau}\right)=\Delta-\sin \left(\theta_{s}+\phi\right)+\chi \sin \left(\phi^{\tau}-\phi-\psi\right)
$$

where we used the shorthand $\phi^{\tau}=\phi(t-\tau)$. Sufficiently far from the kink, the LSs can be approximated by their exponential tails. The latter govern the approach of the steady state. The left and right tails can be expanded as

$$
\begin{aligned}
& \phi(t)=\phi(-\infty)+\sum_{i} a_{-}^{(i)} \exp \left(\sigma_{-}^{(i)} t\right), \\
& \phi(t)=\phi(+\infty)+\sum_{i} a_{+}^{(i)} \exp \left(\sigma_{+}^{(i)} t\right)
\end{aligned}
$$

with the asymptotic values $\phi(-\infty)=0$ and $\phi(+\infty)=2 \pi$ and $\sigma_{ \pm}^{(i)}$ being complex eigenvalues and $a_{ \pm}^{(i)}$ the corresponding eigenvectors. Note that a real-valued $\sigma_{ \pm}^{(i)}$ leads to a monotonic tail while $\sigma_{ \pm}^{(i)} \in \mathbb{C}$ induces oscillatory tails. For the solution to remain bounded, all coefficients $a_{+}^{(i)}$ with $\sigma_{+}^{(i)}>0$ and $a_{-}^{(i)}$ with $\sigma_{-}^{(i)}<0$ have to be zero. If the LSs are sufficiently far away from each other, their interaction is governed by the slowest decaying mode; we denote $\sigma_{+}$(resp. $\sigma_{-}$) the eigenvalues with smallest negative (resp. positive) real part associated with the eigenvector $a_{ \pm}$. As the eigenvalue can be complex, the tail is in general approximated by

$$
\phi(t)=\phi( \pm \infty)+\Re\left(a_{ \pm} e^{\sigma_{ \pm} t}\right) .
$$

Because we are considering periodic solutions with pe$\operatorname{riod} T=\tau+r$, where $r$ is defined as the solution drifts $r=T-\tau$, we can rewrite Eq. (15) as an advanced time-delayed equation ${ }^{34,38}$, replacing $\phi(t-\tau)$ by $\phi(t+r)$. How $T$-periodic solutions approach the uniform state is found by inserting the exponential expansion Eqs. ((17),(18)) into the advanced delayed equation $\dot{\phi}=F\left(\phi, \phi^{-r}\right)$. A linear analysis allows to obtain the equation governed the eigenvalues $\sigma_{ \pm}^{(i)}$ as

$$
\sigma_{ \pm}^{(i)}=A+B \exp \left(\sigma_{ \pm}^{(i)} r\right)
$$

where $A$ and $B$ are the same coefficients as in Eqs. (3) and (4). The exponents of the expansion are therefore the solutions of the eigenvalue problem for the steady states, in which the delay $\tau$ is replaced with a small negative time $r$. Notice that $r$ can only be obtained numerically using direct time integration or during continuation with dde-biftool while the amplitudes $a_{ \pm}$are obtained by a best fit of the tails of the LS using Eq. (19).

To approximate the interaction between two distant LSs located in $x_{1}$ and $x_{2}$ with $x_{1}<x_{2}$, we assume an ansatz

$$
\phi(t)=\phi_{1}(t)+\phi_{2}(t)
$$

where $\phi_{i}(t)=\Phi\left[t-x_{i}(t)\right]$ is a $2 \pi$ kink centered around the position $x_{i}(t)$ and the function $\Phi$ is a $T$-periodic solution that verifies the equation of motion of a single LS, i.e. $\dot{\Phi}=F\left(\Phi, \Phi^{\tau}\right)$. One can expand the left hand side of Eq. (15) and find

$$
\dot{\phi}=\left(1-\dot{x}_{1}\right) \dot{\phi}_{1}-\left(1-\dot{x}_{2}\right) \dot{\phi}_{2} \text {. }
$$

In the vicinity of the second LS one can approximate the right hand side of Eq. (15) as

$$
F\left(\phi, \phi^{\tau}\right) \simeq F\left(\phi_{2}, \phi_{2}^{\tau}\right)+\phi_{1} \frac{\partial F}{\partial \phi}\left(\phi_{2}, \phi_{2}^{\tau}\right)+\phi_{1}^{\tau} \frac{\partial F}{\partial \phi^{\tau}}\left(\phi_{2}, \phi_{2}^{\tau}\right) .
$$

Expressing the instantaneous and delayed tail of $\phi_{1}$ as

$$
\begin{aligned}
& \phi_{1}=\phi\left(t-x_{1}\right)=\Re\left[a_{+} e^{\sigma_{+}\left(t-x_{1}\right)}\right], \\
& \phi_{1}^{\tau}=\phi\left(t+r-x_{1}^{\tau}\right)=\Re\left[a_{+} e^{\sigma_{+}\left(t+r-x_{1}^{\tau}\right)}\right],
\end{aligned}
$$

one can derive the following equation:

$$
\begin{aligned}
-\dot{x}_{1} \dot{\phi}_{1}-\dot{x}_{2} \dot{\phi}_{2} & =\Re\left\{a_{+} e^{\sigma_{+}\left(t-x_{1}\right)}\left[\partial_{1} F\left(\phi_{2}, \phi_{2}^{\tau}\right)-\sigma_{+}\right]\right. \\
& \left.+a_{+} e^{\sigma_{+}\left(t+r-x_{1}^{\tau}\right)} \partial_{2} F\left(\phi_{2}, \phi_{2}^{\tau}\right)\right\} .
\end{aligned}
$$

Before proceeding to the projection of Eq. (25) onto the neutral mode of the adjoint problem, some useful simplifications can be performed by noticing that the displacements $\dot{x}_{i}$ are already small quantities, as their source stem from overlap integrals. As such, in the equation for $\dot{x}_{2}$, the cross inertia term $\dot{x}_{1}$ will be multiplied by an overlap integral $\int \dot{\phi}_{2}^{\dagger} \dot{\phi}_{1}$ which is a small quantity. Similarly, the time delay $x_{1}(t-\tau)$ in Eq. (25) can be expanded to first order in $\tau$, which will generate another contribution proportional to $\dot{x}_{1}$, that can be neglected for the same reason.

For the $T$-periodic solution $\Phi(t)$, the Floquet analysis is obtained setting $\phi(t)=\Phi(t)+\varepsilon u(t)$ which results in a linear delay equation with $T$-periodic coefficients

$$
\dot{u}=a(t) u(t)+b(t) u(t-\tau) .
$$

Performing the linear stability analysis of Eq. 26 yields a neutral eigenfunction with the Floquet multiplier $\mu=1$ and denoted $u_{0}=\dot{\Phi}$ that represents a translation along the periodic orbit. The adjoint problem is defined with 
respect to the standard scalar product of two functions $(u, v)=\int u \bar{v} d t$ and reads

$$
-\dot{v}=a^{\dagger}(t) v(t)+b^{\dagger}(t+\tau) v(t+\tau)
$$

which can be simplified using that $a$ and $b$ are real valued scalar functions for which $\left(a^{\dagger}, b^{\dagger}\right)=(a, b)$. Equation (27) corresponds to delayed equation with a negative delay and $T$-periodic coefficients that must be integrated backward in time. The adjoint problem given by Eq. 27 possesses the same eigenvalues than the direct problem Eq. 26, although the eigenvalue $\mu=1$ is associated to a different eigenvector that we note in the following $v_{0}=\dot{\Phi}^{\dagger}$. We note the eigenvectors of Eq. 26 and of Eq. $27\left\{u_{j}\right\}$ and $\left\{v_{j}\right\}$, respectively. Since these two sets are bi-orthogonal with respect to they other, we have, by a proper normalization choice, $\left(u_{j}, v_{k}\right)=\delta_{j k}$. The motion induced by the tail of a distant LS over another can be calculated by projecting its contribution in Eq. (25) along $v_{0}$. In order to find this particular eigen- function, we partially diagonalized the evolution operator corresponding to Eq. (27) using the Implicitly Restarted Arnoldi Method (IRAM) ${ }^{40}$ with a method similar to the one described in ${ }^{29}$. However, as we are mainly interested in the LS solutions that are stable, the largest multiplier in both the direct and adjoint linearized problem is $\mu=1$. Hence one could evaluate $\dot{\Phi}^{\dagger}$ by simply integrating numerically Eq. (27) starting from a random initial condition until the solution converges towards a periodic profile. Repeating the same analysis in the vicinity of the first LS and using the asymptotic expansion for the second LS that involves $\left(a_{-}, \sigma_{-}\right)$yields the equation of motion for $x_{1}$ and $x_{2}$ as

$$
\begin{aligned}
& \dot{x}_{2}=\Re\left[F_{+} e^{\sigma_{+}\left(x_{2}-x_{1}\right)}\right], \\
& \dot{x}_{1}=\Re\left[F_{-} e^{\sigma_{-}\left(x_{1}-x_{2}\right)}\right],
\end{aligned}
$$

with the coefficients $F_{ \pm}$given by:

$$
F_{ \pm}=\frac{\int_{-\infty}^{\infty} \dot{\Phi}^{\dagger}(s) e^{\sigma_{ \pm} s} \times\left[\frac{\partial F}{\partial \phi}\left(\Phi, \Phi^{\tau}\right)-\sigma_{ \pm}+e^{\sigma_{ \pm} r} \frac{\partial F}{\partial \phi^{\tau}}\left(\Phi, \Phi^{\tau}\right)\right] \mathrm{d} s}{\int_{-\infty}^{\infty} \dot{\Phi}^{\dagger}(s) \dot{\Phi}(s) \mathrm{d} s} a_{ \pm}
$$

The equation of motion for the distance between the two LSs $l=x_{2}-x_{1}$ can be recast as to depend on the gradient of a potential $U(l)$

$$
i=-\frac{d U}{d l}, U(l)=\Re\left(\frac{F_{-}}{\sigma_{-}} e^{-\sigma_{-} l}-\frac{F_{+}}{\sigma_{+}} e^{\sigma_{+} l}\right) .
$$
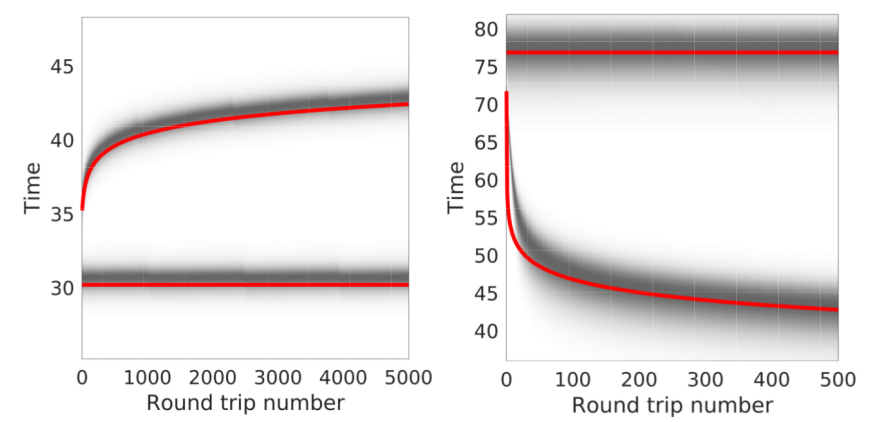

Figure 11. Asymmetrical interactions between LSs. The DNS of the delayed Adler equation Eq. (1) and of the reduced model Eqs. (28),(29) are shown as shades of gray and red lines, respectively. Left: Repulsive "causal" interaction for parameters $(\Delta, \chi, \psi, \tau)=(0.9393,0.99,0,100)$ leading to $\left(F_{+}, F_{-}\right)=(4.88,0.003)$ and $\left(\sigma_{+}, \sigma_{-}\right)=(-0.8,1.21)$. Right: Repulsive "anti-causal" interaction for parameters $(\Delta, \chi, \psi, \tau)=(0.9974,2,1.396,100)$ leading to $\left(F_{+}, F_{-}\right)=$ $\left(5 \times 10^{-4},-4159\right)$ and $\left(\sigma_{+}, \sigma_{-}\right)=(-0.48,0.4)$.

In order to visualize the interaction between the LSs we use a pseudo-space-time representation as introduced
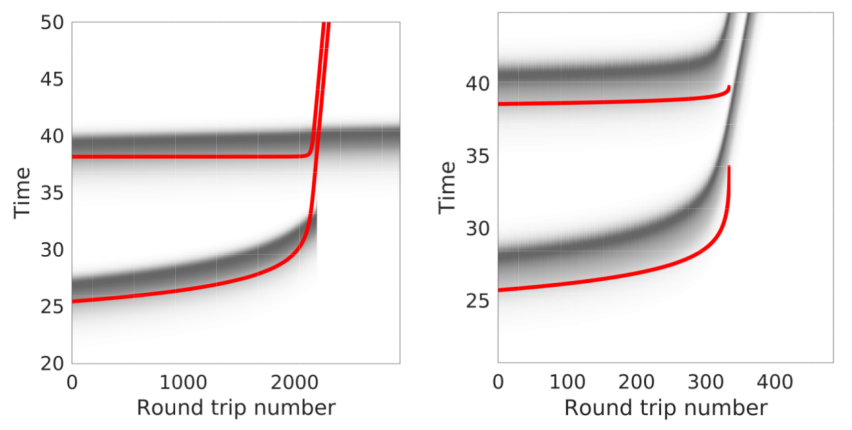

Figure 12. Creation of drifting bound states of LSs. The DNS of the delayed Adler Eq. (1) and of the reduced model Eqs. (28),(29) are shown as shades of gray and red lines, respectively. Left: One of the LSs in the Adler equation disappear while the effective model shows the presence of the bound state. Parameters are $(\Delta, \chi, \psi, \tau)=(0.842,0.99,0.698,100)$ leading to $\left(F_{+}, F_{-}\right)=(341,2)$ and $\left(\sigma_{+}, \sigma_{-}\right)=(-1.7,0.6)$. Right: A molecule is formed in the Adler equation while the effective model give rise to a singularity. Parameters are $(\Delta, \chi, \psi, \tau)=(1.1,0.5,1.323,100)$ leading to $\left(F_{+}, F_{-}\right)=$ $(69,536)$ and $\left(\sigma_{+}, \sigma_{-}\right)=(-0.9,0.92)$.

$\mathrm{in}^{24}$. This is achieved by cutting the time trace in slices of one period $T$ and rearranging them in a second dimension. This is similar to the approach of multiple timescale analysis, since we display the dynamics happening in one period on one axis and show the slow evolution from one 
period to the next on the other axis. One example of such a pseudo-space time representation can be seen in Fig. 11 where we prepared two LSs close to each other as an initial condition. The vertical axis represents the fast time scale and goes from 0 to $T$, yet only the region containing the two LSs is shown. The horizontal axis displays the round-trip number. The results of the direct numerical simulations (DNSs) are displayed in gray scale of the variable $\cos \phi$. In this way, the steady states appear white and the LSs appear in a shade of gray representing their distance to $\theta_{s}$. The red lines overlaying the diagram are the results of an integration of the reduced system given by Eqs. (28),(29).

One can generally observe a good agreement between the results of the reduced equations of motion Eqs. (28),(29) and that of the DNS, as seen for instance in Fig. 11 when the LSs are not too close. The nonreciprocity of the interactions is clearly observed and it is mainly due to the asymmetry of the tails of the LSs and of the overlap integrals; in general $\sigma_{+} \neq-\sigma_{-}$and $F_{+} \neq-F_{-}$. In particular, $F_{+}>0$ (resp. $\left.F_{+}<0\right)$ corresponds to $\mathrm{LS}_{2}$ being repulsed (resp. attracted) by $\mathrm{LS}_{1}$ while and $F_{-}<0$ (resp. and $F_{-}>0$ ) corresponds to $\mathrm{LS}_{1}$ being attracted (resp. repulsed) by $\mathrm{LS}_{1}$. Notice that the left panel situation in Fig. 11 corresponds very well to the experimental results in Figs. 1(c) of ${ }^{30}$. While these results can be reproduced numerically with a Class-C laser model in Fig. 5 of $^{30}$, we show that the interaction are properly accounted for in our simplified Adler model.

The absence of parity make it so that it is possible to obtain stable bound states even in the cases in which $\sigma_{ \pm} \in \mathbb{R}$. We show how non-reciprocal attractive interactions can lead to drifting, stable bound states in Fig. 12. Unfortunately, as the LSs are extremely close to each other, the reduced set of Eqs. (28),(29) diverges in some cases instead of showing a molecule bound state at this parameter set. Yet, other parameter sets allows finding such drifting bound states. For very small distance between the LSs the assumption of weak, single exponential long range interaction is not valid anymore. A possible improvement over Eqs. (28),(29) would be to include higher order terms in the approximation of the tails in Eq. (19) and the projection onto the weakly damped eigenmodes of Eq. 27.

There is a special regime in parameter space, where the exponential tails become complex. This region lies in the vicinity of the $\mathrm{AH}$ instability of the steady states and the tails of the LS starts to oscillate at the frequencies given by $\Im\left(\sigma_{ \pm}\right)$. This opens the possibility for multiple roots for Eq. (31) and thus a potential $U(l)$ with multiples (almost) equidistant minima and maxima. One set of parameters $(\Delta, \chi, \psi, \tau)=(0.49,0.99,2.1,100)$ exhibiting this behavior is displayed in Fig. 13. The corresponding potential $U(l)$ is depicted in Fig. 13(a). The DNS of the Adler Eq. (1) with two LSs at varying distances was performed and Fig. 13(c) shows the evolution of the distance between the LSs over multiple round-trips. The maxima and minima of the potential are shown in red
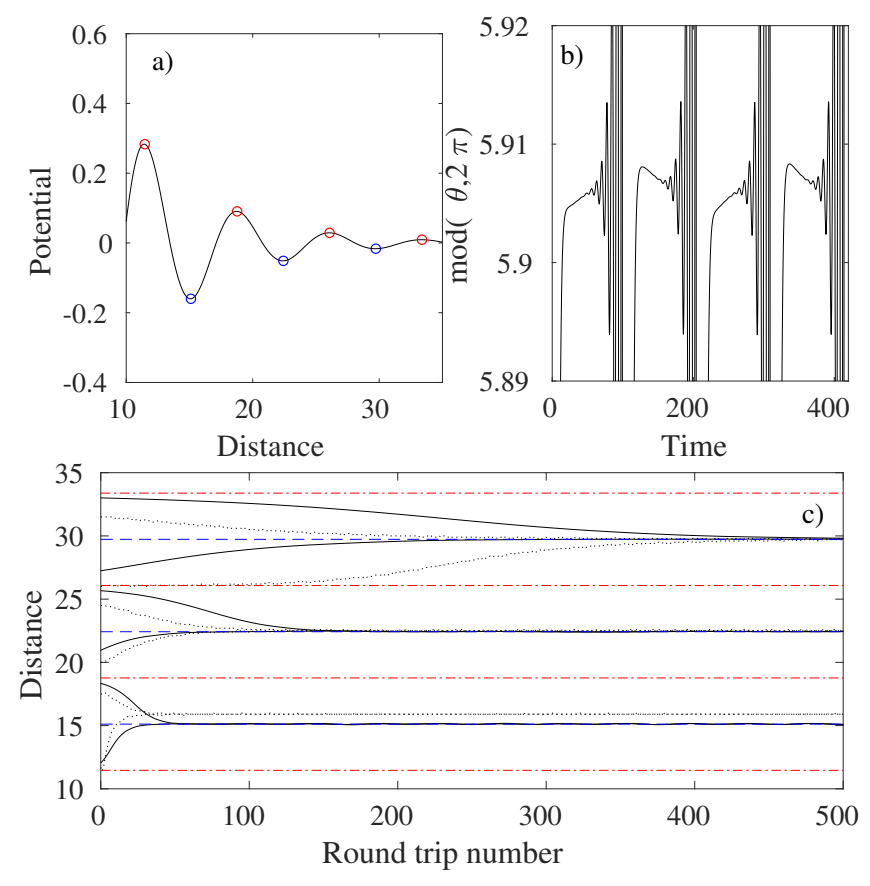

Figure 13. (a) Potential of the distance between two LSs. (b) DNS of the Adler Eq. (1) showing a precursor of a periodtwo instability of a single LS. (c) Evolution of the distance between two LSs obtained from DNS of Adler Eq. (1) in dotted black lines and reduced model Eq. (28),(29) in solid black lines as well as minima and maxima of the potential in blue dashed and red dot-dashed lines. Parameters are $(\Delta, \chi, \psi, \tau)=(0.49,0.99,2.1,100)$ in all three cases.

and blue dashed lines, respectively while the distances obtained from the reduced Eqs. (28)(29) are displayed in black lines. The distance obtained from DNS is also shown in dotted lines. The results of the DNS do not seem to match very well with that of the reduced model in this particular case. While converging to the same distances after many round-trips the transients are different. The reason for the discrepancy was found in the pathological values of the parameters we chose for the periodic solution; The feedback phases $\psi>\pi / 2$ bring the system close to a period doubling regime. A precursor of that period doubling instability can be observed in the DNS of a single LS which is shown in Fig. 13(b). The time trace is zoomed into the vicinity of the steady state $\theta_{s}$ to show the small deviation between the relaxations into the steady state happening every other period. It is well-known that period-two oscillations of LSs can modify strongly their interactions, up to the point of canceling the coarsening dynamics as shown in $^{39}$.

In the following we will combine some of the results obtained in the previous sections to show how one could manipulate a system of multiple LSs by changing the systems parameters. We start the simulation in the regime where LSs can form a molecule bound state, if they are close enough $(\Delta, \chi, \psi, \tau)=(1.1,0.99,1.7,100)$ as seen in Fig. 11(b), with five LSs at varying starting 


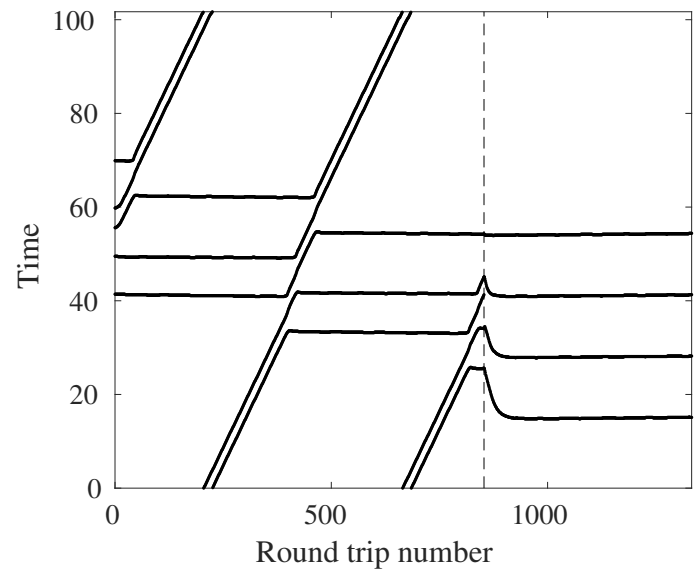

Figure 14. Pseudo space-time representation of the positions of multiple LSs obtained by DNSs of the Adler Eq. (1). The simulation starts with five LSs at varying distances. Parameters are $\Delta=1.1, \chi=1.7, \chi=0.99$ and $\tau=100$. The parameters change at the dashed line to $\Delta=0.6, \psi=2.1$, $\chi=0.99$ and $\tau=100$.

distances. Two of the LSs are close enough to form a molecule bound state that starts to drift. Figure 14 shows the DNS in pseudo-space-time. Upon reaching another LS the molecule bound state splits in two while one of the LSs forms another molecule bound state with the newly encountered LS. Since the domain is periodic this molecule bound state moves trough it repetitively and interacts with every other LSs in the delay line. This dynamics is in good agreement with the experimental results of Fig. 3 of $^{30}$. After round trip 850 (indicated by a dashed line) the system parameters were changed to $(\Delta, \chi, \psi, \tau)=(0.6,0.99,2.1,100)$ which is close to the parameters in Fig. 13 and leads to the same regular locking behavior. One can clearly see that the LSs organize themselves in an equidistant pattern since every LS locks the next one into a minimum of the induced potential. However there are only four LSs on the right side of the dashed line while the system started with five LSs prior to the parameter change. The annihilation of one LS is most likely caused by the abrupt change of parameters, combined with the small distance with neighbors.

\section{CONCLUSION}

The time-delayed Adler equation is a prototypical model for the dynamics of the phase of an injected semiconductor laser with coherent injection and delayed feedback. It consists in a single, $2 \pi$ periodic degree of freedom and of only three control parameters. It is arguably one of the simplest model giving rise to topological localized structures. In this paper we studied the bifurcation mechanisms that govern the stability of the locked solution and the appearance of stable LSs. We have found that the locked solution can become unstable via saddle- node bifurcations, as in the standard, not delayed Adler equation, but also via Andronov-Hopf bifurcations, which is a direct consequence of the presence of the time delay. In the long delay limit, approximations of the $\mathrm{SN}$ and $\mathrm{AH}$ borders were given using the quasi-continuous spectrum method. We have found that the branches of single and multiple LSs are usually connected and that parameter sweeps induce transitions between states with different numbers of evenly spaced LSs. The branches of LSs were found to emerge either from diverging period solutions or from saddle-node of limit cycle bifurcations. Finally, we provided the derivation of the effective equations of motion governing the distance between LSs. We have found that the leading eigenvalue expansions can be obtained solving a linear, time-advanced, equation and that the lack of parity leads to non-reciprocal interactions and drifting bound states. Finally, we observed how the transition from real towards complex eigenvalues explains the creation of stable bound states. Further works would consider the statistical dynamics of a large ensemble of LSs.

\section{ACKNOWLEDGMENTS}

J.J. acknowledge the financial support of the MINECO Project MOVELIGHT (PGC2018-099637-B100 AEI/FEDER UE). S.G. acknowledges the PRIME program of the German Academic Exchange Service (DAAD) with funds from the German Federal Ministry of Education and Research (BMBF).

\section{REFERENCES}

${ }^{1}$ J. Wu, R. Keolian, and I. Rudnick. Observation of a nonpropagating hydrodynamic soliton. Phys. Rev. Lett., 52:1421-1424, Apr 1984.

${ }^{2}$ E. Moses, J. Fineberg, and V. Steinberg. Multistability and confined traveling-wave patterns in a convecting binary mixture. Phys. Rev. A, 35:2757-2760, Mar 1987.

${ }^{3}$ F. J. Niedernostheide, M. Arps, R. Dohmen, H. Willebrand, and H. G. Purwins. Spatial and spatio-temporal patterns in pnpn semiconductor devices. physica status solidi (b), 172(1):249-266, 1992.

${ }^{4}$ P. B. Umbanhowar, F. Melo, and H. L. Swinney. Localized excitations in a vertically vibrated granular layer. Nature, (382):793$796,1996$.

${ }^{5}$ Yuri A. Astrov and H.G. Purwins. Plasma spots in a gas discharge system: birth, scattering and formation of molecules. Physics Letters A, 283(5-6):349 - 354, 2001.

${ }^{6} \mathrm{G}$. Nicolis and I. Prigogine. Self-Organization in Nonequilibrium Systems: From Dissipative Structures to Order through Fluctuations. Wiley, 1977.

${ }^{7}$ L.A. Lugiato. Transverse nonlinear optics: Introduction and review. Chaos, Solitons and Fractals, 4(8-9):1251 - 1258, 1994. Special Issue: Nonlinear Optical Structures, Patterns, Chaos.

${ }^{8} \mathrm{P}$. Mandel and M. Tlidi. Transverse dynamics in cavity nonlinear optics (2000-2003). Journal of Optics B: Quantum and Semiclassical Optics, 6(9):R60, 2004.

${ }^{9}$ T. Ackemann, W. J. Firth, and G.L. Oppo. Chapter 6 fundamentals and applications of spatial dissipative solitons in photonic devices. In P. R. Berman E. Arimondo and C. C. Lin, editors, 
Advances in Atomic Molecular and Optical Physics, volume 57 of Advances In Atomic, Molecular, and Optical Physics, pages 323 - 421. Academic Press, 2009.

${ }^{10}$ W. J. Firth and A. J. Scroggie. Optical bullet holes: Robust controllable localized states of a nonlinear cavity. Phys. Rev. Lett., 76:1623-1626, Mar 1996.

${ }^{11}$ M. Brambilla, L. A. Lugiato, F. Prati, L. Spinelli, and W. J. Firth. Spatial soliton pixels in semiconductor devices. Phys. Rev. Lett., 79:2042-2045, 1997.

${ }^{12}$ S. Barland, J. R. Tredicce, M. Brambilla, L. A. Lugiato, S. Balle, M. Giudici, T. Maggipinto, L. Spinelli, G. Tissoni, T. Knödl, M. Miller, and R. Jäger. Cavity solitons as pixels in semiconductor microcavities. Nature, 419(6908):699-702, Oct 2002.

${ }^{13}$ F. Leo, S. Coen, P. Kockaert, S.P. Gorza, P. Emplit, and M. Haelterman. Temporal cavity solitons in one-dimensional Kerr media as bits in an all-optical buffer. Nat Photon, 4(7):471-476, Jul 2010.

${ }^{14}$ T. Herr, V. Brasch, J. D. Jost, C. Y. Wang, N. M. Kondratiev, M. L. Gorodetsky, and T. J. Kippenberg. Temporal solitons in optical microresonators. Nature Photonics, 8(2):145-152, 2014.

${ }^{15}$ N. N. Rosanov and G. V. Khodova. Autosolitons in nonlinear interferometers. Opt. Spectrosc., 65:449-450, 1988.

${ }^{16}$ N. N. Rosanov and G. V. Khodova. Diffractive autosolitons in nonlinear interferometers. J. Opt. Soc. Am. B, 7:1057-1065, 1990.

${ }^{17}$ P. Genevet, S. Barland, M. Giudici, and J. R. Tredicce. Bistable and addressable localized vortices in semiconductor lasers. Phys. Rev. Lett., 104:223902, Jun 2010.

${ }^{18}$ Y. Tanguy, T. Ackemann, W. J. Firth, and R. Jäger. Realization of a semiconductor-based cavity soliton laser. Phys. Rev. Lett., 100:013907, Jan 2008.

${ }^{19}$ M. Marconi, J. Javaloyes, S. Balle, and M. Giudici. How lasing localized structures evolve out of passive mode locking. Phys. Rev. Lett., 112:223901, Jun 2014.

${ }^{20}$ J. Javaloyes. Cavity light bullets in passively mode-locked semiconductor lasers. Phys. Rev. Lett., 116:043901, Jan 2016.

${ }^{21}$ J. Javaloyes, P. Camelin, M. Marconi, and M. Giudici. Dynamics of localized structures in systems with broken parity symmetry. Phys. Rev. Lett., 116:133901, Mar 2016.

${ }^{22}$ P. Camelin, J. Javaloyes, M. Marconi, and M. Giudici. Electrical addressing and temporal tweezing of localized pulses in passivelymode-locked semiconductor lasers. Phys. Rev. A, 94:063854, Dec 2016.

${ }^{23}$ F. T. Arecchi, G. Giacomelli, A. Lapucci, and R. Meucci. Twodimensional representation of a delayed dynamical system. Phys. Rev. A, 45:R4225-R4228, Apr 1992.

${ }^{24}$ G. Giacomelli and A. Politi. Relationship between delayed and spatially extended dynamical systems. Phys. Rev. Lett., 76:2686-
2689, Apr 1996

${ }^{25}$ S.A. Kashchenko. The Ginzburg-Landau equation as a normal form for a second-order difference-differential equation with a large delay. Comput. Math. Math. Phys., 38(3):1, 1998.

${ }^{26}$ Serhiy Yanchuk and Giovanni Giacomelli. Spatio-temporal phenomena in complex systems with time delays. Journal of Physics A: Mathematical and Theoretical, 50(10):103001, 2017.

${ }^{27}$ B. Romeira, R. Avó, José M. L. Figueiredo, S. Barland, and J. Javaloyes. Regenerative memory in time-delayed neuromorphic photonic resonators. Scientific Reports, 6:19510 EP -, Jan 2016. Article.

${ }^{28}$ M. Marconi, J. Javaloyes, S. Barland, S. Balle, and M. Giudici. Vectorial dissipative solitons in vertical-cavity surface-emitting lasers with delays. Nature Photonics, 9:450-455, 2015.

${ }^{29}$ B. Garbin, J. Javaloyes, G. Tissoni, and S. Barland. Topological solitons as addressable phase bits in a driven laser. Nat. Com., 6, 2015.

${ }^{30}$ B. Garbin, J. Javaloyes, S. Barland, and G. Tissoni. Interactions and collisions of topological solitons in a semiconductor laser with optical injection and feedback. Chaos: An Interdisciplinary Journal of Nonlinear Science, 27(11):114308, 2017.

${ }^{31}$ K. Engelborghs, T. Luzyanina, and D. Roose. Numerical bifurcation analysis of delay differential equations using dde-biftool. ACM Trans. Math. Softw., 28(1):1-21, March 2002.

${ }^{32}$ B. Garbin, A. Dolcemascolo, F. Prati, J. Javaloyes, G. Tissoni, and S. Barland. Refractory period of an excitable semiconductor laser with optical injection. Phys. Rev. E, 95:012214, Jan 2017.

${ }^{33}$ P. Coullet, D. Daboussy, and J. R. Tredicce. Optical excitable waves. Phys. Rev. E, 58:5347-5350, Nov 1998.

${ }^{34}$ Serhiy Yanchuk, Stefan Ruschel, Jan Sieber, and Matthias Wolfrum. Temporal dissipative solitons in time-delay feedback systems. Phys. Rev. Lett., 123:053901, Jul 2019.

${ }^{35}$ R. M. Corless, G. H. Gonnet, D. E. G. Hare, D. J. Jeffrey, and D. E. Knuth. On the lambertw function. Advances in Computational Mathematics, 5(1):329-359, Dec 1996.

${ }^{36}$ Serhiy Yanchuk, Leonhard Lücken, Matthias Wolfrum, and Alexander Mielke. Spectrum and amplitude equations for scalar delay-differential equations with large delay. Discrete and Continuous Dynamical Systems, 35(1):537-553, 2015.

${ }^{37}$ Steven H. Strogatz. Nonlinear Dynamics and Chaos. Westview Press, 2015.

${ }^{38}$ Michel Nizette. Stability of square oscillations in a delayedfeedback system. Phys. Rev. E, 70:056204, Nov 2004.

${ }^{39}$ J. Javaloyes, T. Ackemann, and A. Hurtado. Arrest of domain coarsening via anti-periodic regimes in delay systems. Phys. Rev. Lett., 115:223901, Nov 2015.

${ }^{40}$ R. Lehoucq and D. Sorensen. Deflation techniques for an implicitly restarted arnoldi iteration. SIAM Journal on Matrix Analysis and Applications, 17(4):789-821, 1996. 